H. NAKAGAWA

KODAI MATH. J.

3 (1980), 321-340

\title{
ON A CERTAIN MINIMAL IMMERSION OF A RIEMANNIAN MANIFOLD INTO A SPHERE
}

\author{
By Hisao NAKAgawa
}

Dedicated to Professor I. Mogi on his 60th birthday

Introduction. Minimal immersions of spheres into a sphere have been completely determined by do Carmo and Wallach [2]. Let $H^{r, n}$ be the space of all spherical harmonic polynomials of degree $r$ on an $n$-dimensional sphere $S^{n}$, where $\operatorname{dim} H^{r, n}=(n+2 r-1)(n+r-2) ! / r !(n-1) !=: N(r)+1$. For an orthonormal basis $\left\{f_{1}, \cdots, f_{N(r)+1}\right\}$ of $H^{r, n}$, we define an immersion $\iota_{r}$ of $S^{n}$ into an $(N(r)+1)-$ dimensional Euclidean space $\boldsymbol{R}^{N(r)+1}$ by $\iota_{r}(x)=\left(f_{1}(x), \cdots, f_{N(r)+1}\right) /(N(r)+1)^{1 / 2}$, which is called a standard immersion. Then the image by $c_{r}$ is contained in the unit sphere $S^{N(r)}(1)$ in $\boldsymbol{R}^{N(r)+1}$, and by means of a theorem of Takahashi [2] it is seen that $\iota_{r}$ is a minimal isometric immersion and $\iota_{r}\left(S^{n}\right)$ is not contained in the great sphere of $S^{N(r)}(1)$. With regard to the degree of the immersion in the sense of Wallach [9], they showed that the degree of $\iota_{r}$ is equal to $r$ and if $r \leqq 3$, then $\iota_{r}$ is rigid.

On the other hand, Hong [3] introduced recently a notion of planar geodesic immersions. Let $M$ and $\tilde{M}$ be complete connected Riemannian manifolds of dimension $n$ and $n+p$, respectively. An isometric immersion c of $M$ into $\tilde{M}$ is called a planar geodesic immersion if each geodesic in $M$ is mapped locally under the immersion into a 2-dimensional totally geodesic submanifold of $\tilde{M}$. Planar geodesic immersions of $M$ into $S^{n+p}(c)$ have been classified by Little [5] and Sakamoto [8], independently, who stated that $M$ is a compact symmetric space of rank one and the second fundamental form is parallel. The so-called Veronese manifold can be considered as one of examples determined by the planar geodesic immersion, while it can be regarded as the case of degree 2 in the ambient space.

When one pays attension to the rigidness of the standard immersion $c_{r}$, it seems to be important to study the structure of the immersion with lower degree. As a matter of fact, the local version and the characterization of the Veronese manifold which is essentially an easiest model in our situation are investigated from variously different viewpoints. Furthermore, the local version concerning the immersion $\iota_{3}$ of $S^{n}$ into $S^{N(3)}$ has been treated by the author and Itoh [6]. In this paper, we shall be concerned with the characterization of the standard immersion $\iota_{3}$ of $S^{n}$ into $S^{N(3)}$. So as to do so, the notion of planar geodesic

Received May 12, 1979 
immersions is generalized. The purpose of this paper is to prove Theorem 8.1.

The author would like to express his hearty thanks to Professor T. Itoh for his suggestions and advise during the preparation of this paper.

1. Preliminaries. First of all, we recall the general theory of Riemannian submanifolds immersed into a Riemannian manifold to fix our notation and state several properties which are subsequently useful. We denote by $M^{n}(c)$ an $n(\geqq 3)$ dimensional connected Riemannian manifold of constant curvature $c$, which is called a real space form of constant curvature $c$. Let $M$ be an $n(\geqq 3)$-dimensional connected and orientable Riemannian manifold with the Riemannian metric $g$ and let c be an isometric immersion of $M$ into $\tilde{M}=M^{n+p}(c)$. When the argument is only considered in the local version, $M$ need not be distinguished from $c(M)$. So, in order to simplify the discussion, we identify any point $x$ in $M$ with the point $\iota(x)$, and moreover any tangent vector $u$ at $x$ with the tangent vector $d \iota_{x}(u)$.

Now we choose an orthonormal local frame field $\left\{e_{1}, \cdots, e_{n}, e_{n+1}, \cdots, e_{n+p}\right\}$ of $\tilde{M}$ in such a way that, restricted to $M$, the vectors $e_{1}, \cdots, e_{n}$ are tangent to $M$ and hence the others are normal to $M$. Restricting the canonical forms and the connection forms on $\tilde{M}$ with respect to these frames to $M$, we denote them by $\omega_{A}, \omega_{A B}$, where $A, B=1, \cdots, n, n+1, \cdots, n+p$. We then have

$$
\omega_{\alpha}=0 \text {. }
$$

Here and in the sequel, we make use of the following convention on the range of indices:

$$
\begin{aligned}
& \imath, \jmath, \cdots=1, \cdots, n, \\
& \alpha, \beta, \cdots=n+1, \cdots, n+p .
\end{aligned}
$$

The metric induced from the Riemannian metric in the ambient space $\tilde{M}$ under the immersion $c$ is given by $g=2 \sum_{i} \omega_{i} \cdot \omega_{i}$. Then $\left\{e_{1}, \cdots, e_{n}\right\}$ are also the orthonormal frame field with respect to the induced metric, and $\left\{\omega_{1}, \cdots, \omega_{n}\right\}$ are the dual field with respect to $\left\{e_{1}, \cdots, e_{n}\right\}$. It follows from (1.1) and Cartan's lemma that we have

$$
\omega_{\alpha \imath}=\sum_{\jmath} h_{\imath j}^{\alpha} \omega_{j}, \quad h_{\imath \jmath}^{\alpha}=h_{j i}^{\alpha} .
$$

The quadratic form $\Sigma_{\imath, j} h_{\imath \jmath}^{\alpha} \omega_{i} \omega_{j}$ is called the second fundamental form of the immersion $c$ on $M$ in the direction of $e_{\alpha}$. At any point $x$ of $M$, we denote by $X$ and $Y$ (resp. $\xi$ and $\eta$ ) vector fields on a neighborhood of $x$ tangent (resp. normal) to $M$. The second fundamental form $\sigma$ of $M$ can be written as

$$
\sigma(X, Y)=\sum_{\alpha, \imath, j} h_{\imath j}^{\alpha} \omega_{i}(X) \omega_{j}(Y) e_{\alpha}
$$

for any vector fields $X$ and $Y$. By means of the structure equations of the ambient space, we have the following structure equations of the submanifold $M$ :

$$
d \omega_{i}+\sum_{\jmath} \omega_{i \jmath} \wedge \omega_{j}=0, \quad \omega_{i j}+\omega_{j i}=0,
$$




$$
\begin{array}{r}
d \omega_{i \jmath}+\sum_{k} \omega_{i k} \wedge \omega_{k \jmath}=\Omega_{\imath \jmath}, \\
\Omega_{\imath j}=-\frac{1}{2} \sum_{k, l} R_{\imath \jmath k l} \omega_{k} \wedge \omega_{l},
\end{array}
$$

where $\omega_{i \jmath}$ (resp. $\Omega_{\imath \jmath}$ ) denotes the connection form (resp. the curvature form) on $M$. Moreover, we have

$$
d \omega_{\alpha \beta}+\sum_{r} \omega_{\alpha \gamma} \wedge \omega_{\gamma \beta}=\Omega_{\alpha \beta}, \quad \Omega_{\alpha \beta}=-\frac{1}{2} \sum_{k, l} R_{\alpha \beta k l} \omega_{k} \wedge \omega_{l},
$$

where $\omega_{\alpha \beta}$ defines the connection induced in the normal bundle on $M$ and $\Omega_{\alpha \beta}$ is called the normal curvature form on $M$. From (1.2) and (1.5) we have the equation of Gauss

$$
R_{\imath j k l}=c\left(\delta_{i l} \delta_{j k}-\delta_{i k} \delta_{\jmath l}\right)+\sum_{\alpha}\left(h_{\imath l}^{\alpha} h_{j k}^{\alpha}-h_{i k}^{\alpha} h_{j l}^{\alpha}\right) .
$$

For the second fundamental form $\sigma$ the vector $\sigma\left(e_{2}, e_{2}\right)$ is called a normal curvature vector in the direction of a unit vector $e_{\imath}$. If every normal curvature vector has the same length for any unit vector $u$ at $x$, then the immersion $c$ is said to be isotropic at $x$. If $c$ is isotropic at any point on $M$, namely, the length of a normal curvature vector depends only on the initial point, then the immersion is said to be isotropic. In particular, if the length is equal to $\lambda$, the immersion is said to be $\lambda$-isotropic, and the isotropy $\lambda$ is a continuous function defined on $M$, the square of which is smooth. The immersion $\iota$ is $\lambda$-isotropic at a point $x$ if and only if the second fundamental form satisfies

$$
\widetilde{S}_{3}\left\langle\sigma\left(u_{1}, u_{2}\right), \sigma\left(u_{3}, v\right)\right\rangle=\lambda^{2} \widetilde{S}_{3}\left\langle u_{1}, u_{2}\right\rangle\left\langle u_{3}, v\right\rangle,
$$

where $u_{\imath}(\imath=1,2,3)$ and $v$ are unit vectors at $x$ and $\varsigma_{m}$ denotes the cyclic sum with respect to vectors $u_{1}, \cdots, u_{m}$. The condition is equivalent to

$$
\langle\sigma(u, u), \sigma(u, v)\rangle=0
$$

for any orthonormal vectors $u$ and $v$ at $x$ [7].

Now, we denote by $\nabla$ (resp. $\tilde{\nabla}$ ) the covariant differentiation on $M$ (resp. $\tilde{M}$ ). For any vector fields $X$ and $Y=\Sigma_{\imath} y_{i} e_{\imath}$, we have

$$
\begin{aligned}
& \nabla_{X} Y=\sum_{k}\left\{d y_{k}(X)-\sum_{\imath} y_{i} \omega_{i k}(X)\right\} e_{k}, \\
& \tilde{\nabla}_{X} Y=\nabla_{X} Y+\sigma(X, Y)=\nabla_{X} Y+\sum_{\alpha, \imath, \jmath} h_{\imath \jmath}^{\alpha} \omega_{i}(X) \omega_{j}(Y) e_{\alpha} .
\end{aligned}
$$

For any vector fields $\xi$ and $X$ on a neighborhood of $x$ in $M$, we may express

$$
\tilde{\nabla}_{X} \xi=-H_{\xi} X+\nabla_{X}^{\perp} \xi
$$

where $-H_{\xi} X$ and $\nabla_{X}^{\frac{1}{X}} \xi$ denote the components of $\tilde{\nabla}_{X} \xi$ tangent and normal to $M$, 
respectively. Then $\nabla_{X}^{\frac{1}{X}}$ becomes the covariant differentiation with respect to the induced connection in the normal bundle $N(M)$. The tangent component $H_{\xi} X$ is related to the second fundamental form $\sigma$ as follows: $\left\langle H_{\xi} X, Y\right\rangle=\langle\sigma(X, Y), \xi\rangle$ for any vector field $Y$. Then $H_{\xi}$ is a symmetric transformation of the tangent space of $M$. In particular, we have

$$
\tilde{\nabla}_{X} e_{\alpha}=-\sum_{k} \omega_{\alpha k}(X) e_{k}-\sum_{\beta} \omega_{\alpha \beta}(X) e_{\beta} .
$$

We define the covariant derivative $h_{\imath j k}^{\alpha}$ of $h_{\imath \jmath}^{\alpha}$ by

$$
\sum_{k} h_{\imath j_{k}}^{\alpha} \omega_{k}=d h_{\imath \jmath}^{\alpha}-\sum_{l} h_{l j}^{\alpha} \omega_{i l}-\sum_{l} h_{i l}^{\alpha} \omega_{l \jmath}-\sum_{\beta} h_{\imath j}^{\beta} \omega_{\beta \alpha} .
$$

Since $\tilde{M}$ is of constant curvature, we get

$$
h_{\imath j k}^{\alpha}-h_{i k J}^{\alpha}=0 .
$$

Now, suppose that $h_{\imath_{1} \cdots \imath_{m}}^{\alpha}$ are defined for some $m \geqq 2$. Inductively we define the covariant derivative $h_{\imath_{1} \cdots \imath_{m} \jmath}^{\alpha}$ of $h_{\imath_{1} \cdots \imath_{m}}^{\alpha}$ by

$(1.15)_{m} \quad \sum_{\jmath} h_{\imath_{1} \cdots \imath_{m} \jmath}^{\alpha} \omega_{\jmath}=d h_{\imath_{1} \cdots \imath_{m}}^{\alpha}-\sum_{r=1}^{m} \sum_{\jmath} h_{\imath_{1} \cdots \imath_{r-1} j i_{r+1} \cdots \imath_{m}}^{\alpha} \omega_{j i_{r}}-\sum_{\beta} h_{i_{1} \cdots \imath_{m}}^{\beta} \omega_{\beta \alpha}$.

From the equation above we have the following Ricci formula:

$$
\begin{aligned}
& h_{\imath_{1} \cdots \imath_{m} j k}^{\alpha}-h_{\imath_{1} \cdots \imath_{m} k_{j}}^{\alpha} \\
= & c \sum_{r=1}^{m}\left(h_{\imath_{1} \cdots \imath_{r-1} j i_{r+1} \cdots \imath_{m}}^{\alpha} \delta_{\imath_{r} k}-h_{\imath_{1} \cdots \imath_{r-1}{ }^{k} \imath_{r+1} \cdots \imath_{m}}^{\alpha} \delta_{\imath_{r j}}\right) \\
& +\sum_{r=1}^{m} \sum_{\beta, l} h_{\imath_{1} \cdots \imath_{r-1} \imath_{r} \imath_{r+1} \cdots \imath_{m}}^{\alpha}\left(h_{i_{r k}}^{\beta} h_{l j}^{\beta}-h_{i_{r} j}^{\beta} h_{l k}^{\beta}\right) \\
& +\sum_{\beta, l} h_{i_{1} \cdots \imath_{m}}^{\beta}\left(h_{l k}^{\alpha} h_{l j}^{\beta}-h_{l j}^{\alpha} h_{l k}^{\beta}\right) .
\end{aligned}
$$

We define the covariant differentiation $\nabla^{\prime}$ on the Whitney sum $T(M) \oplus N(M)$ as follows: For any $N(M)$-valued tensor field $T$ of type $(0, k)$, we define

$$
\left(\nabla_{X}^{\prime} T\right)\left(Y_{1}, \cdots, Y_{k}\right)=\nabla_{X}^{\frac{1}{X}}\left(T\left(Y_{1}, \cdots, Y_{k}\right)\right)-\sum_{r=1}^{k} T\left(Y_{1}, \cdots, \nabla_{X} Y_{r}, \cdots, Y_{k}\right)
$$

and $\nabla^{\prime} T$ ia also defined by $\left(\nabla^{\prime} T\right)\left(Y_{1}, \cdots, Y_{k}, X\right)=\left(\nabla_{X}^{\prime} T\right)\left(Y_{1}, \cdots Y_{k}\right)$, which is an $N(M)$-valued tensor field of type $(0, k+1)$. We denote by $\nabla^{\prime 2} T$ the covariant derivative of $\nabla^{\prime} T$. Furthermore, we can inductively define a covariant derivative $\nabla^{\prime m} T$. Then, for the second fundamental form $\sigma$, it follows

$$
\begin{aligned}
& \left(\nabla^{\prime m} \sigma\right)\left(Y, Z, X_{1}, \cdots, X_{m}\right) \\
& \quad=\sum_{\alpha, \jmath, k, \imath_{1}, \cdots, \imath_{m}} h_{j \ell_{1} \cdots \imath_{m}}^{\alpha} \omega_{j}(Y) \omega_{k}(Z) \omega_{i_{1}}\left(X_{1}\right) \cdots \omega_{i_{m}}\left(X_{m}\right) e_{\alpha} .
\end{aligned}
$$


2. Degrees of isometric immersions. In this section, let c be an isometric immersion of $M$ into $\tilde{M}=M^{n+p}(c)$. We consider the decomposition of the normal bundle of the immersion. For any point $x$ in $M$ the normal space of $M$ is simply denoted by $N_{x}$. Namely, we have $N_{x}=\left(d \iota_{x}\left(M_{x}\right)\right)^{N}$, where $(.)^{N}$ means taking the orthogonal complement into the tangent space $T_{\iota(x)}(\tilde{M})$ in the ambient space. The second fundamental form $\sigma$ of $\iota$ at $x$ is a bilinear map of $M_{x} \times M_{x}$ into $N_{x}$ defined by $\sigma(X, Y)=\left(\tilde{\nabla}_{X} Y\right)^{N}$, where $X$ and $Y$ are vector fields on a neighborhood of $x$ in $M$. For convenience' sake, we put $\sigma_{2 x}=\sigma_{x}$, and so $\sigma_{2 x}$ can be regarded as a linear map of a symmetric square $S^{2}\left(M_{x}\right)$ of $M_{x}$ into the normal space $N_{x}$. We set $N_{x}^{1}:=\sigma_{2 x}\left(S^{2}\left(M_{x}\right)\right)$, which is called a first normal space of $\iota$ at $x$. This means that $N_{x}^{1}$ is the linear subspace of $N_{x}$ spanned by normal vectors $\sigma(u, v)$ for any vectors $u$ and $v$ at $x$, so we see that $\operatorname{dim} N_{x}^{1} \leqq{ }_{n} H_{2}=n(n+1) / 2$. In particular, if $\iota$ is minimal, then $\operatorname{dim} N_{x}^{1} \leqq n(n+1) / 2-1$. The point $x$ of $M$ is said to be 2-regular if $N_{x}^{1}$ is of maximal dimension with respect to basic points. Let $M_{2}$ be the set consisting of all 2-regular points of $M$. Then $M_{2}$ is open in $M$. For any point $x$ in $M_{2}$, we set $O_{x}^{2}:=d \iota_{x}\left(M_{x}\right) \oplus N_{x}^{1}$, which is called the second osculating space of $c$ at $x$. Now, for the 2-regular point $x$ we define a trilinear map $\sigma_{3 x}$ of $M_{x} \times M_{x} \times M_{x}$ into $\left(O_{x}^{2}\right)^{N}$ by $\sigma_{3 x}(X, Y, Z):=\left(\tilde{\nabla}_{X}\left(\sigma_{2 x}(Y, Z)\right)\right)^{N_{2}}$ for any tangent vecor fields $X, Y$ and $Z$, where $(.)^{N_{2}}$ denotes the orthogonal projection into $\left(O_{x}^{2}\right)^{N} . \quad \sigma_{3 x}$ is well defined and it is symmetric, because $\tilde{M}$ is of constant curvature, so it induces a linear map $\sigma_{3 x}: S^{3}\left(M_{x}\right) \rightarrow\left(O_{x}^{2}\right)^{N}$ of the symmetric third power of $M_{x}$ into $\left(O_{x}^{2}\right)^{N}$. We call $\sigma_{3 x}$ the third fundamental form of $c$ at $x$ and the linear subspace $N_{x}^{2}:=\sigma_{3 x}\left(S^{3}\left(M_{x}\right)\right)$ is called the second normal space of c at $x$. The second normal space $N_{x}^{2}$ at $x$ is the orthogonal projection of the linear subspace spanned by $\nabla^{\prime} \sigma(v, w, u)$ for any vectors $u, v$ and $w$ at $x$, so $\operatorname{dim} N_{x}^{2} \leqq{ }_{n} H_{3}$ $=n(n+1)(n+2) / 6$. In particular, if $\iota$ is minimal, then $\operatorname{dim} N_{x}^{2} \leqq n(n+1)(n+2) / 6-n$. The point $x$ in $M_{2}$ is said to be 3-regular if $N_{x}^{2}$ is of maximal dimension with respect to basic points. Let $M_{3}$ be the set consisting of all 3-regular points of $M_{2}$. Then $M_{3}$ is also open in $M_{2}$. We put $O_{x}^{3}:=O_{x}^{2} \oplus N_{x}^{2}$, which is called the third osculating space of $c$ at $x$.

We now proceed inductively and suppoese that the $(\jmath-1)$-th osculating space $O_{x}^{\jmath-1}$ of $c$ at the $(\jmath-1)$-regular point $x$ is defined. Then we see that it is possible to define a linear map $\sigma_{\jmath x}$ of the symmetric $\jmath$-th power of $M_{x}$ into $\left(O_{x}^{3-1}\right)^{N}$ by

$$
\sigma_{\jmath x}\left(X_{1}, \cdots, X_{j}\right):=\left(\tilde{\nabla}_{X_{1}}\left(\sigma_{\jmath-1 x}\left(X_{2}, \cdots, X_{\jmath}\right)\right)\right)^{N_{\jmath-\imath}}
$$

for any vector fields $X_{1}, \cdots, X_{\jmath}$, where $(.)^{N_{\jmath-1}}$ denotes the orthogonal projection into $\left(O_{x}^{\jmath-1}\right)^{N}$. We call $\sigma_{\jmath x}$ the $j$-th fundamental form of $\iota$ at $x, N_{x}^{\jmath-1}:=\sigma_{\jmath x}\left(S^{j}\left(M_{x}\right)\right)$ the $(j-1)$-th normal space of $c$ at $x$, and $O_{x}^{\prime}:=O_{x}^{j-1} \oplus N_{x}^{j-1}$ the $j$-th osculating space of $c$ at $x$. Clearly, the process must be eventually stopped, because of $\operatorname{dim} O_{x}^{\jmath} \leqq \operatorname{dim} \tilde{M}$. Thus there exists a first integer $q$ for which $\sigma_{\jmath}=0$ for $\jmath>q$ and $\sigma_{q} \neq 0$. Then $q$ is called the degree of $\iota$ and the set $M_{q}$ is open in $M$.

We shall be here concerned with the decomposition above of Riemannian homogeneous spaces and then standard immersions of Riemannian symmetric spaces into a sphere. Let $M=G / K$ be a Riemannian homogeneous space, where 
$G$ is a compact connected Lie group which acts effectively on $M$ and $K$ is a closed subgroup of $G$, and let $\langle$,$\rangle be the G$-invariant Riemannian metric on $M$. The isometric immersion c of $M$ into $\tilde{M}$ is said to be equvvalent, if there is a continuous group homomorphism $\rho: G \rightarrow I(\tilde{M})$ such that $\iota(g x)=\rho(g) \iota(x)$ for any element $g$ in $G$ and any point $x$ in $M$, where $I(\tilde{M})$ denotes the group of isometries of $\tilde{M}$. Assume that $\iota$ is equivalent. It follows from the equvalence of $\checkmark$ that the dimension of the $\jmath$-th normal space $N_{x}^{j}$ is independent of the basic point $x$, so $M_{\text {J }}$ coincides with $M$ itself. We put $N_{x}^{0}=d \iota_{x}\left(M_{x}\right)$ for convenience' sake. This gives a decomposition of the restriction $T_{M}(\tilde{M})$ to $\iota(M)$ of the tangent bundle $T(\tilde{M})$ of $\tilde{M}$ into the Whitney sum $T_{M}(\tilde{M})=\sum_{j=0}^{q-1} N^{j} \oplus\left(O^{q}\right)^{N}$, where the fiber of $N^{j}$ at $x$ is $N_{x}^{j}$ and the fiber of $\left(O^{q}\right)^{N}$ at $x$ is $\left(O_{x}^{q}\right)^{N}$. Let $o$ be the origin of $M=G / K$. The linear isotropy representation is the homomorphism of $K$ into the group of linear transformations of $T_{o}(M)$ which assign to each $k \in K$ the differential of $k$ at $o$. We denote by $K^{*}$ the image of $K$ by the linear isotropy representation, which is called the linear isotropy group at $o$. Then we remark that the action of $K^{*}$ on $M_{o}=T_{o}(M)$ may be extended to $S^{j}\left(M_{o}\right)$ by the usual tensor action. Consequently, we have $\sigma_{\jmath}(k u)=k \sigma_{j}(u)$ for any element $u$ in $S^{j}\left(M_{o}\right), k$ in $K$, and $\jmath=2, \cdots, q$, by means of the equivalence of $\iota$. This shows that we have $\left\|\sigma_{2}(u, u)\right\|=\left\|\sigma_{2}(k u, k u)\right\|$ for any vector $u$ in $M_{o}$. Thus the immersion $c$ is constant isotropic, because of the equivalence of $\iota$.

In particular, let $M=G / K$ be a compact symmetric space where the isotropy aciton of $K$ is irreducible, and let $\Delta$ be the Laplace operator for $(M,\langle\rangle$,$) , where$ $\langle$,$\rangle is some G$-invariant Riemannian structure up to scalar multilple. For any $\lambda \in R$, let $V_{\lambda}$ be the eigenspace with the eigenvalue $\lambda$ of $\Delta$, and for any real valued functions $f_{1}$ and $f_{2}$ on $M$, let $\left(f_{1}, f_{2}\right):=\int_{M} f_{1} f_{2} d M$. Then $V_{\lambda}$ is a vector space over $R$ endowed with the inner product (,). Let $p_{\lambda}+1:=\operatorname{dim} V_{\lambda}$. For each non-zero eigenvalue $\lambda$, let $f_{1}, \cdots f_{p_{\lambda}+1}$ be an orthonormal basis of $V_{\lambda}$. We define $\iota_{\lambda}: M \rightarrow R^{p_{\lambda}+1}$ by

$$
\iota_{\lambda}(x)=\left(f_{1}(x), \cdots, f_{p_{\lambda}+1}(x)\right) /\left(p_{\lambda}+1\right)^{1 / 2} .
$$

Then it is seen in [9] that $c_{\lambda}$ is a minimal isometric immersion of $(M,\langle\rangle$,$) into$ a $p_{\lambda}$-dimensional sphere $S^{p_{\lambda}(1)}$ of constant curvature 1 . We call such $c_{\lambda}$ the standard immersion of $M$. By a theorem of Wallach [9], if the degree of the standard immersion $c_{\lambda}$ is less than or equal to 3 , then $c_{\lambda}$ is rigid. Evidently, a rigid immersion of a homogeneous space is equivalent.

3. Cubic geodesic immersions. Let $c$ be an isotropic immersion of an $n$ -dimensional Riemannian manifold $M$ into an $(n+p)$-dimensional real space form $\tilde{M}=M^{n+p}(c)$ of constant curvature $c$, where $p \geqq 2$. For a regular curve $C:(a, b)$ $\rightarrow M$, if there exist an open interval $I_{t}$ of each $t$ in $(a, b)$ and a 3-dimensional totally geodesic submanifold $M_{t}$ in $\tilde{M}$ such that $I_{t} \subset(a, b)$ and $\iota\left(C\left(I_{t}\right)\right) \subset M_{t}$, then the curve is said to be locally cubrc. The immersion $c$ is called a cubic geodesic 
ımmersion, if any geodesic in $M$ is locally cubic. This can be regarded as the generalization of the concept of planar geodesic immersions, though it seems to be quite probable that isotropicness is the superfluous condition. In particular, a cubic geodesic immersion which is not planar geodesic is said to be proper.

Now, we replace isotropicness in the condition of cubic geodesic immersions with another one related much more to cubic curves themselves. From now on, we assume that the isometric immersion of $M$ into $\tilde{M}=M^{n+p}(c)$ satisfies only the condition that each geodesic on $M$ is locally a cubic curve. For any point $x$ and any unit vector $u$ at $x$, let $\gamma_{u}$ be a geodesic in $M$ passing through $x=\gamma_{u}(0)$ with the initial vector $\gamma_{u}^{\prime}(0)=u$ and parametrized by arc length $s$, which is called a normal geodesic. Let $X(s)=\gamma_{u}^{\prime}(s)$. Then (1.11) is reduced to

$$
\tilde{\nabla}_{X} Y=\nabla_{X} Y+\sigma(X, Y)=\nabla_{X} Y+\sum_{\alpha, \imath, \jmath} h_{\imath \jmath}^{\alpha} \omega_{\imath}(X) \omega_{j}(Y) e_{\alpha} .
$$

It follows from (1.12) and (1.17) that we have

$$
\begin{aligned}
\widetilde{\nabla}_{X}(\sigma(Y, Z))= & \left(\nabla^{\prime} \sigma\right)(Y, Z, X)+\sigma\left(\nabla_{X} Y, Z\right)+\sigma\left(Y, \nabla_{X} Y\right) \\
& -\sum_{\imath}\left\langle\sigma(Y, Z), \sigma\left(X, e_{\imath}\right)\right\rangle e_{\imath}
\end{aligned}
$$

for any vector fields $Y$ and $Z$ along $\gamma_{u}$ tangent to $M$. Since $\gamma_{u}$ is locally contained in a 3-dimensional totally geodesic submanifold $M_{0}$ in $\tilde{M}$, local vector fields $X, \sigma(X, X)$ and $\tilde{\nabla}_{X}(\sigma(X, X))$ are tangent to $M_{o}$ at $\gamma_{u}(s)$ for a sufficiently small $s$.

LEMMA 3.1. The immersion is cubic geodesic if and only if the orthogonal complement of the vector $\gamma_{u}^{\prime}(0)$ in the tangent space $T_{x} M_{0}$ is a subspace of the normal space $N_{x}$ for any point $x$ and any unt vector $u$ at $x$.

Proof. We suppose that the orthogonal complement of $T_{x} M_{0}$ is contained in $N_{x}$ and then it suffices to show that $\iota$ is isotropic. We may suppose that $\sigma(u, u) \neq 0$ for a unit vector $u$. For a normal geodesic $\gamma_{u}:(-a, a) \rightarrow M$, there is a sufficiently small interval $I$ of 0 in $(-a, a)$ such that ${ }^{\circ} \circ \gamma_{u} \mid I$ is contained in a 3 -dimensional totally geodesic submanifold $M_{o}$ and moreover we have $\sigma(X, X) \neq 0$, where $X(s)=\gamma_{u}^{\prime}(s)$. Accordingly, there exists a unit vector $\xi_{1}$ along $\gamma_{u} \mid I$ such that $\sigma(X, X)=k \xi_{1}$, where $k(s)=\|\sigma(X, X)\|$ is a positive smooth function. Since $\tilde{\nabla}_{X}(\sigma(X, X))$ is tangent to $M_{o}$, it is spanned by three mutually orthogonal vector fields $X, \xi_{1}$ and $\xi$ along $\gamma_{u} \mid I$. Namely,

$$
\tilde{\nabla}_{X}(\sigma(X, X))=f X+g \xi_{1}+\xi,
$$

where $f$ and $g$ are smooth functions. It follows from (3.2), (3.3) and the assumption that we have $\langle\sigma(X, X), \sigma(X, Y)\rangle+f\langle X, Y\rangle=0$ along $\gamma_{u} \mid I$, which yields $\langle\sigma(u, u), \sigma(u, v)\rangle=0$ for any orthonormal vectors $u$ and $v$ at $x$. Thus $\iota$ is isotropic, because of (1.9). 
Conversely, since $\iota$ is $\lambda$-isotropic, it follows from (3.2) that (3.3) is reduced to $\nabla^{\prime} \sigma(X, X, X)-\lambda^{2} X=f X+g \xi_{1}+\xi$, which implies $\langle\xi, Y\rangle=0$ for any vector field $Y$ orthogonal to $X$. Therefore $\xi$ is normal to $M$.

We shall here give an important example of a proper cubic geodesic immersion. We are concerned with the standard immersion $\iota_{3}$ of $S^{n}(1)$ into $S^{N(3)}(c)$ mentioned in the introduction, where $N(3)+1=(n+5)(n+1) ! / 3 !(n-1) !$ and $c=n / 3(n+2)$. Then it is seen in $[2,9]$ that the degree of $c_{3}$ is equal to 3 . For orthonormal elements $u$ and $v$ of $S^{n}(1)$ in $\boldsymbol{R}^{n+1}$, we identify $v$ with a unit tangent vector at $u$. Then $\gamma: t \rightarrow u \cos t+v \sin t$ is a geodesic in $S^{n}(1)$ with the initial vector $v$ and moreover, by the construction of the immersion, we have

$$
\iota_{3}(\gamma(t))=u_{1} \cos t+v_{1} \sin t+u_{3} \cos 3 t+v_{3} \sin 3 t,
$$

where $u_{\imath}$ and $v_{\imath}(\imath=1,3)$ are fixed elements in $\boldsymbol{R}^{N(3)+1}$. This means that the curve $c_{3} \circ \gamma$ is contained in the 4-dimensional linear subspace spanned by the vectors $u_{\imath}$ and $v_{\imath}$ in $\boldsymbol{R}^{N(3)+1}$, and therefore every geodesic in $S^{n}(1)$ is mapped in a 3-dimensional totally geodesic submanifold of $S^{N(3)}(c)$ under the immersion $\iota_{3}$. It implies that $\iota_{3}$ is properly cubic geodesic.

4. Properties of cubic geodesic immersions. In this section, let $c$ be a cubic geodesic immersion of $M$ into $\tilde{M}=M^{n+p}(c)$. By using notations in the previous section, general properties of cubic geodesic immersions are studied. We denote by $\kappa(s)$ the isotropy at the point $\gamma_{u}(s)$. Namely, $k(s)=\|\sigma(X, X)\|$, the square of which is a smooth function along $\gamma_{u}$. We suppose that $k$ is positive. Then $k$ is also smooth and there exists a unit vector field $\xi_{1}$ along $\gamma_{u}$ normal to $M$ such that

$$
\sigma(X, X)=k \xi_{1} .
$$

Since $\tilde{\nabla}_{X} \xi_{1}$ is also tangent to $M_{o}$, there is a vector field $\xi$ normal to $M$ and mutually orthogonal to $X$ and $\xi_{1}$ such that

$$
\tilde{\nabla}_{X} \xi_{1}=-k X+\xi \text {. }
$$

By means of (3.2), (4.1) and (4.2) we have

$$
(X k) \xi_{1}+k \xi=\nabla^{\prime} \sigma(X, X, X),
$$

because $\iota$ is $k$-isotropic. By virtue of (1.12) and (1.17), we get

$$
\begin{aligned}
\tilde{\nabla}_{X}\left(\nabla^{\prime} \sigma(Y, Z, W)\right)= & \nabla^{\prime 2} \sigma(Y, Z, W, X)+\nabla^{\prime} \sigma\left(\nabla_{X} Y, Z, W\right) \\
& +\nabla^{\prime} \sigma\left(Y, \nabla_{X} Z, W\right)+\nabla^{\prime} \sigma\left(Y, Z, \nabla_{X} W\right) \\
& -\sum_{\imath}\left\langle\nabla^{\prime} \sigma(Y, Z, W), \sigma\left(X, e_{\imath}\right)\right\rangle e_{\imath} .
\end{aligned}
$$

Differentiating (4.3) in the direction of $X$ and using (4.4), we have 


$$
\begin{aligned}
& -k(X k) X+\left(X^{2} k\right) \xi_{1}+2(X k) \xi+k \tilde{\nabla}_{X} \xi \\
& =\nabla^{\prime 2} \sigma(X, X, X, X)-\sum_{\imath}\left\langle\nabla^{\prime} \sigma(X, X, X), \sigma\left(X, e_{\imath}\right)\right\rangle e_{\imath} .
\end{aligned}
$$

Since $X, \xi_{1}$ and $\xi$ are mutually orthogonal, $\tilde{\nabla}_{X} \xi$ is orthogonal to $X$, and therefore it is only spanned by $\xi_{1}$ and $\xi$, because $\tilde{\nabla}_{X} \xi$ is also tangent to $M_{o}$. It implies together with (4.5) that we have

$$
2\left\langle\nabla^{\prime} \sigma(X, X, X), \sigma(X, Y)\right\rangle=\left(X k^{2}\right)\langle X, Y\rangle .
$$

Making use of this equation, we have

\section{LEMMA 4.1. i is constant isotropic.}

Proof. We may suppose that there is a point $x$ such that the isotropy $k$ at the point is positive. Then we can choose a normal coordinate neighborhood $U$ of $x$ in $M$ such that $k>0$ on $U$. For any unit vectors $u$ and $v$ at $x$, let $\gamma_{u}$ and $\gamma_{v}$ be normal geodesics in $U$ passing through $x=\gamma_{u}(0)=\gamma_{v}(0)$. Let $X=\gamma_{u}^{\prime}$ and $Y=\gamma_{v}^{\prime}$. Differentiating $\langle\sigma(X, X), \sigma(X, Z)\rangle=k^{2}\langle X, Z\rangle$ obtained by (1.8) in the direction of $X$ and using (3.2), we have $\left\langle\nabla^{\prime} \sigma(X, X, X), \sigma(X, Z)\right\rangle+\langle\sigma(X, X)$, $\left.\nabla^{\prime} \sigma(X, X, Z)\right\rangle=\left(X k^{2}\right)\langle X, Z\rangle$ for any vector field $Z$ along $\gamma_{u}$, because $\nabla^{\prime} \sigma$ is symmetric. On the other hand, differentiating $\|\sigma(W, W)\|^{2}=k^{2}$ in the direction of $Y$, we have $2\left\langle\sigma(W, W), \nabla^{\prime} \sigma(W, W, Y)\right\rangle=Y k^{2}$ for any vector field $W$ along $\gamma_{v}$. Combining these two equations with (4.6), we have $Y k^{2}=\left(X k^{2}\right)\langle u, v\rangle$ at the point $x$. This means that $k^{2}$ is constant along any geodesic $\gamma_{v}$, and therefore it is constant on $U$. Consequently, if there exists a point $x$ such that $k(x)>0$, then $k$ is constant on $M$.

Q.E. D.

By means of this lemma, we may assume that the isotropy $k$ is positive. Now, taking account of (4.2), we see that $c$ is the planar geodesic immersion, provided that $\xi$ vanishes identically. Therefore, we may suppose that there exist a point $x$ and a unit vector $u$ at $x$ so that the normal vector field $\xi$ defined by (4.2) is not a zero vector at $x$. It follows from the continuity of $\xi$ that we may locally suppose that $\xi$ has no zero points along the geodesic passing through $x$ with the direction of $u$. We put $l=\|\xi\|$ and $\xi_{2}=\xi / l$. Then the function $l$ is smooth along $\gamma_{u}$, and it is easily seen that $X, \xi_{1}$ and $\xi_{2}$ are the Frenet frames for $\gamma_{u}$ which satisfy the Frenet formulas. Making use of (4.3), (4.5) and (4.6), we have

$$
\begin{aligned}
& k l \xi_{2}=\nabla^{\prime} \sigma(X, X, X), \\
& \left\langle\nabla^{\prime} \sigma(X, X, X), \sigma(X, Y)\right\rangle=0, \\
& l^{3} \sigma(X, X)-(X l) \nabla^{\prime} \sigma(X, X, X)+l \nabla^{\prime 2} \sigma(X, X, X, X)=0
\end{aligned}
$$

for any vector field $Y$ along $\gamma_{u}$. Moreover, since $c$ is constant isotropic, we have

$$
\left\langle\sigma(X, X), \nabla^{\prime} \sigma(X, X, Y)\right\rangle=0 \text {. }
$$


For any fixed point $x$ we can regard $l$ as the function on the tangent unit sphere $S_{x}$ at $x$. Concerning this function, we find

\section{LEMMA 4.2. $l$ is independent of any unit vector at $x$.}

Proof. We suppose that there exists a unit vector $u$ in $S_{x}$ such that $l(u)>0$. We take a normal coordinate neighborhood $U$ of $x$. Differentiating (4.8) in the direction of $X$ and making use of (4.4), (4.8) and (4.9), we get

$$
\left\langle\nabla^{\prime} \sigma(X, X, X), \nabla^{\prime} \sigma(X, X, Y)\right\rangle=(k l)^{2}\langle X, Y\rangle
$$

for any vector field $Y$.

In spite of the value of the function $l$, as a direct consequence of (4.3) and the equation above, we have $\left\langle\nabla^{\prime} \sigma(v, v, v), \nabla^{\prime} \sigma(v, v, w)\right\rangle=0$ for any orthonormal vectors $v$ and $w$. We put $v_{1}=a v+b w, w_{1}=b v-a w$ and $v_{2}=b v+a w, w_{2}=a v-b w$, where $a$ and $b$ are real numbers such that $a^{2}+b^{2}=1$. Then pairs $\left(v_{1}, w_{1}\right)$ and $\left(v_{2}, w_{2}\right)$ are both orthonormal, and so we have $\left\langle\nabla^{\prime} \sigma\left(v_{\imath}, v_{\imath}, v_{\imath}\right), \nabla^{\prime} \sigma\left(v_{\imath}, v_{\imath}, w_{\imath}\right)\right\rangle=0$ $(i=1,2)$. Summing up these equations, we obtain

$$
\begin{aligned}
a b\left(a^{4}+b^{4}\right) & \left(\left\|\nabla^{\prime} \sigma(v, v, v)\right\|^{2}-\left\|\nabla^{\prime} \sigma(w, w, w)\right\|^{2}\right)-a b\left(a^{4}-4 a^{2} b^{2}+b^{4}\right) \\
& \cdot\left\{3\left(\left\|\nabla^{\prime} \sigma(v, v, w)\right\|^{2}-\left\|\nabla^{\prime} \sigma(v, w, w)\right\|^{2}\right)+2\left(\left\langle\nabla^{\prime} \sigma(v, v, v), \nabla^{\prime} \sigma(v, w, w)\right\rangle\right.\right. \\
- & \left.\left.\left\langle\nabla^{\prime} \sigma(w, w, w), \nabla^{\prime} \sigma(w, v, v)\right\rangle\right)\right\}=0 .
\end{aligned}
$$

If we put $a^{2}=(3+\sqrt{3}) / 6$ and $b^{2}=(3-\sqrt{3}) / 6$, then this yields $\left\|\nabla^{\prime} \sigma(v, v, v)\right\|^{2}=$ $\left\|\nabla^{\prime} \sigma(w, w, w)\right\|^{2}$ By means of (4.3) and (4.7), we have $(k l(v))^{2}=(k l(w))^{2}$, which means that

$$
l(v)=l(w) .
$$

For any vector $v$ in $S_{x}$, let $V(v)$ be a subset in $S_{x}$ consisting of vectors $w$ such that $l(v)=l(w)$, and let $L$ be a subspace of $M_{x}$ spanned by $e_{2}, \cdots, e_{n}$ for any orthonormal basis $\left\{e_{1}, e_{2}, \cdots, e_{n}\right\}$ in $M_{x}$ such that $e_{1}=v$. Then (4.12) shows that the intersection of $L$ and $S_{x}$ is contained in $V(v)$. For any vector $w$ in $S_{x}$ which does not belong to $L$, there exist two orthonormal vectors $v_{1}$ and $v_{2}$ in $L$ such that $w=a v_{1}+b v_{2}$, because of $\operatorname{dim} M \geqq 3$. Since $w$ is also orthogonal to $v_{2}, l(w)$ $=l\left(v_{2}\right)$, and therefore $l\left(v_{2}\right)=l(v)$. Thus we see that $w$ belongs to $V(v)$, which means that the subset $V(v)$ coincides with $S_{x}$ itself. Accordingly, the function $l$ is constant on $S_{x}$.

Q.E. D.

Now, let $\phi$ be an $m$-form on a vector space $V$. For any vectors $v_{1}, \cdots, v_{m}$ and any permutation $\tau$ in a symmetric group $S_{m}$ of order $m$, we put $\tau \phi\left(v_{1}, \cdots\right.$, $\left.v_{m}\right)=\phi\left(v_{\tau(1), \ldots,} v_{\tau(m)}\right)$ and we define the symmetrizer $\mathcal{S}_{m}$ by $\mathcal{S}_{m} \phi=\sum_{\tau \in S_{m}} \tau \phi$. By means of Lemma 4.2, (4.7) is true for any unit vector at a fixed point $x$, and therefore it follows from (4.7), (4.8), (4.10) and the linearity of the forms $\sigma$ and $\nabla^{\prime} \sigma$ that we have the following equations at $x$;

$$
\mathcal{S}_{6}\left\langle\nabla^{\prime} \sigma\left(u_{1}, u_{2}, u_{3}\right), \nabla^{\prime} \sigma\left(u_{4}, u_{5}, u_{6}\right)\right\rangle=(k l)^{2} \mathcal{S}_{6}\left\langle u_{1}, u_{2}\right\rangle\left\langle u_{3}, u_{4}\right\rangle\left\langle u_{5}, u_{6}\right\rangle,
$$




$$
\begin{aligned}
& \mathcal{S}_{4}\left\langle\nabla^{\prime} \sigma\left(u_{1}, u_{2}, u_{3}\right), \sigma\left(u_{4}, v\right)\right\rangle=0, \\
& \mathcal{S}_{4}\left\langle\sigma\left(u_{1}, u_{2}\right), \nabla^{\prime} \sigma\left(u_{3}, u_{4}, v\right)\right\rangle=0 .
\end{aligned}
$$

Since $\nabla^{\prime} \sigma$ is symmetric with respect to all elements, $(4.8)^{\prime}$ is reduced to

$$
\widetilde{S}_{4}\left\langle\nabla^{\prime} \sigma\left(u_{1}, u_{2}, u_{3}\right), \sigma\left(u_{4}, v\right)\right\rangle=0 .
$$

Combining $(4.10)^{\prime}$ with (4.8)", we have

$$
\left\langle\sigma(v, w), \nabla^{\prime} \sigma\left(u_{1}, u_{2}, u_{3}\right)\right\rangle=\Im_{3}\left\langle\nabla^{\prime} \sigma\left(v, w, u_{1}\right), \sigma\left(u_{2}, u_{3}\right)\right\rangle .
$$

We define here a helix in an $m$-dimensional Riemannian manifold $N$. Let $C: c=c(s)$ be a regular curve in $N$, where $s$ is the arc length. The curve $C$ is said to be of order $r(\leqq m)$, if it has the Frenet frames $\left\{c(s), \xi_{0}, \cdots, \xi_{r}\right\}$ along $C$ and the following Frenet formulas along $C$ are satisfied:

$$
\left\{\begin{array}{l}
\frac{d c}{d s}=\xi_{0}=: X, \\
\nabla_{X} \xi_{\jmath}=-k_{j}(s) \xi_{\jmath-1}+k_{j+1}(s) \xi_{j+1}
\end{array}\right.
$$

for $\jmath=0, \cdots, r$, where $\nabla$ denotes the covariant differentiation in $N$ and $k_{o}(s)=k_{\jmath+1}$ $(s)=0$ and $k_{j}(s)(\jmath=1, \cdots, r)$ is positive along $C$. Then $k_{j}(s)\left(\right.$ resp. $\left.\xi_{j}\right)$ is called the $j$-th curvature (resp. the $j$-th normal vector) of $C$. For example, a geodesic is a curve of order 0 and, a circle is a curve of order 1 and moreover the first curvature $k_{1}$ is constant. A curve $C$ is called a helıx if $C$ is of order 2 and $k_{1}$ and $k_{2}$ are both constant along $C$. In particular, $\xi_{1}$ is called a prencipal vector and $\xi_{2}$ is called a binormal vector. Let $C: c=c(s)$ be a helix. Then the components satisfy a system of differential equations, because of the Frenet formulas for $C$. According to the fundamental theory of differential equations, we see that there exists a unique solution satisfying the given initial condition in a sufficiently small interval of $s=0$. Namely, for any point $p$ in $N$ and any orthonormal vectors $u, v$ and $w$ at $p$, there exists locally a helix passing through $p$ with a tangent vector $u$, which satisfies certain conditions.

Now, coming back to our situation, it follows from (4.1) and the Frenet formulas that any geodesic $\gamma_{u}$ in $M$ is at most of order 2 , and $k$ and $l$ are the first and second curvature of $\gamma_{u}$, respectively. Moreover, $k$ is constant along $\gamma_{u}$. Concerning with geodesics in $M$, we have

Proposition 4.3. If a cubic geodesic immersion c of $M$ into $\tilde{M}=M^{n+p}(c)$ is proper, then any geodesic in $M$ is a helix in the ambient space $\tilde{M}$.

Proof. In order to prove this property, it suffices to show that the second curvature $l$ along any geodesic in $M$ is positive constant. Since $c$ is not planar geodesic, we may suppose that there exists a point $x$ such that $l(x)>0$. For any unit vectors $u$ and $v$ at $x$, let $\gamma_{u}$ and $\gamma_{v}$ be normal geodesics passing through 
$x=\gamma_{u}(0)=\gamma_{v}(0)$ with the tangent vector $u=\gamma_{u}^{\prime}(0), v=\gamma_{v}^{\prime}(0)$, respectively. Then we take a normal coordinate neighborhood $U$ of $x$ in $M$, on which $l$ is positive. Differentiating (4.11) in the direction of $X=\gamma_{u}^{\prime}$, we have

$$
\begin{aligned}
& \left\langle\nabla^{\prime 2} \sigma(X, X, X, X), \nabla^{\prime} \sigma(X, X, Y)\right\rangle+\left\langle\nabla^{\prime} \sigma(X, X, X), \nabla^{\prime 2} \sigma(X, X, Y, X)\right\rangle \\
& =k^{2}\left(X l^{2}\right)\langle X, Y\rangle .
\end{aligned}
$$

Again, differentiating $(4.7)^{\prime}$ with $u_{\imath}=W(\imath=1, \cdots, 6)$ in the direction of $Z=\gamma_{v}^{\prime}$, we get

$$
2\left\langle\nabla^{\prime} \sigma(W, W, W), \nabla^{\prime 2} \sigma(W, W, W, Z)\right\rangle=k^{2}\left(Z l^{2}\right) .
$$

Since the Ricci formula $(1.16)_{2}$ shows $\nabla^{\prime 2} \sigma(W, W, W, Z)-\nabla^{\prime 2} \sigma(W, W, Z, W)$ can be expressed as a linear combination of vectors $\sigma(W, \quad)$, the above equations and (4.8) give

$$
2\left\langle\nabla^{\prime 2} \sigma(u, u, u, u), \nabla^{\prime} \sigma(u, u, v)\right\rangle=k^{2}\left(2 X l^{2}\langle u, v\rangle-Z l^{2}\right)
$$

at $x$.

On the other hand, combining with (4.9), (4.10) and (4.11), we have $l^{2}\left\langle\nabla^{\prime 2} \sigma(u, u, u, u), \nabla^{\prime} \sigma(u, u, v)\right\rangle=(k l)^{2} X l^{2}\langle u, v\rangle / 2$ at $x$. Thus we see

$$
(k l)^{2}\left(X l^{2}\langle u, v\rangle-Z l^{2}\right)=0,
$$

which implies $l$ is constant along $\gamma_{v}$. This means that we can prove the fact that the second curvature is constant on $U$. The assertion is thereby proved.

Q.E.D.

Remark. Helices in a Riemannian manifold are studied by Ikawa [4].

5. Degrees of cubic geodesic immersions. As is stated in $\S 3$, it is seen that the degree of the standard immersion $\iota_{3}$ of $S^{n}(1)$ into $S^{n+p}(c)$ is equal to 3 . In this section, we shall be concerned with degrees of cubic geodesic immersions of $M$ into $\tilde{M}=M^{n+p}(c)$. First of all, let $c$ be only an isometric immersion of $M$ into $\tilde{M}$, and we assume that every normal goedesic $\gamma: c=c(s)$ in $M$ is a helix in the ambient space. Then the Frenet formulas for $\gamma$ are given by

$$
\left\{\begin{array}{l}
\frac{d c}{d s}=: X, \\
\tilde{\nabla}_{X} X=k \xi_{1}, \\
\tilde{\nabla}_{X} \xi_{1}=-k X+l \xi_{2}, \\
\tilde{\nabla}_{X} \xi_{2}=-l \xi_{1},
\end{array}\right.
$$

where $\left(c(s), X, \xi_{1}, \xi_{2}\right)$ is the Frenet frames for $\gamma$ and $k$ and $l$ are positive constant along $\gamma$. It follows from (1.11) and the second equation of (5.1) that we have $\sigma(X, X)=k \xi_{1}$. Furthermore, by means of (3.2), this equation and the third 
equation of (5.1), we get

$$
-k^{2} X+k l \xi_{2}=\nabla^{\prime} \sigma(X, X, X)-\sum_{\imath}\left\langle\sigma(X, X), \sigma\left(X, e_{\imath}\right)\right\rangle e_{\imath} .
$$

LEMMA 5.1. The immersion i is isotropic if and only if the binormal vector $\xi_{2}$ is normal to $M$.

Proof. If the immersion $c$ is isotropic, then the property of $\sigma(X, X)$ implies that the isotropy of $c$ is equal to $k$. Then it follows from (1.8) that we have $k^{2} X=\sum_{i}\left\langle\sigma(X, X), \sigma\left(X, e_{\imath}\right)\right\rangle e_{\imath}$, because $X$ is a unit vector field along $\gamma$, from which implies, together with (5.2), that we have $\nabla^{\prime} \sigma(X, X, X)=k l \xi_{2}$. Consequently, $\xi_{2}$ is normal to $M$.

Conversely, if $\xi_{2}$ is normal to $M$, then (5.2) yields $\langle\sigma(u, u), \sigma(u, v)\rangle=0$ for any orthonormal vectors $u$ and $v$ at any point $x$, which asserts that the immersion $\iota$ is isotropic.

Q.E. D.

We suppose that $\iota$ is a $k$-isotropic immersion. For a normal geodesic $\gamma_{u}$ passing through $x=r_{u}(0)$ we can make use of many equations obtained in the preceding section, where $k$ and $l$ are positive constant along $\gamma_{u}$. Thus, (4.9) means

$$
\nabla^{\prime 2} \sigma(X, X, X, X)+l^{2} \sigma(X, X)=0
$$

along $\gamma_{u}$. This means that we have $\nabla^{\prime 2} \sigma(u, u, u, u)+l^{2} \sigma(u, u)=0$ for any unit vector $u$ at $x$, so we see $\mathcal{S}_{4}\left\{\nabla^{\prime 2} \sigma\left(u_{1}, u_{2}, u_{3}, u_{4}\right)+l^{2}\left\langle u_{1}, u_{2}\right\rangle \sigma\left(u_{3}, u_{4}\right)\right\}=0$, because the forms $\nabla^{\prime 2} \sigma$ and $\sigma$ are both linear. By taking account of the fact that the form $\nabla^{\prime 2}$ is symmetric with respect to the first three elements, the equation is reduced to

$$
3 ! \Im_{4} \nabla^{\prime 2} \sigma\left(u_{1}, u_{2}, u_{3}, u_{4}\right)+(2 !)^{2} l^{2} \Im_{3}\left\{\left\langle u_{4}, u_{1}\right\rangle \sigma\left(u_{2}, u_{3}\right)+\left\langle u_{1}, u_{2}\right\rangle \sigma\left(u_{3}, u_{4}\right)\right\}=0,
$$

which gives, together with the property of isotropic immersions and the Ricci formula $(1.16)_{2}$,

$$
\begin{aligned}
& 6 \nabla^{\prime 2} \sigma\left(u_{1}, u_{2}, u_{3}, v\right)=\Im_{3}\left\{\left(-3 c+3 k^{2}-l^{2}\right)\left\langle u_{1}, u_{2}\right\rangle \sigma\left(u_{3}, v\right)\right. \\
& \left.\quad+\left(3 c+3 k^{2}-l^{2}\right)\left\langle v, u_{1}\right\rangle \sigma\left(u_{2}, u_{3}\right)-6 \sum_{\imath}\left\langle\sigma\left(e_{\imath}, v\right), \sigma\left(u_{1}, u_{2}\right)\right\rangle \sigma\left(e_{\imath}, u_{3}\right)\right\} .
\end{aligned}
$$

This shows that the 4-th fundamental form vanishes identically on $M$. In other words, the third normal space $N_{x}^{3}$ is trivial. Thus, by the definition of the degree of the immersion, (5.4) says the degree of $c$ is not greater than 3 . On the other hand, by (5.2), $\nabla^{\prime} \sigma(u, u, u)$ is proportional to the binormal vector $\xi_{2}$ which is orthogonal to $\sigma(u, u)=k \xi_{1}$. Consequently, the second normal space $N_{x}^{2}$ is not empty, which proves the following

Proposition 5.2. Let $c$ be an isotropic immersion of $M$ into $\tilde{M}=M^{n+p}(c)$. If any geodesic in $M$ is a helix on $\tilde{M}$, then the degree of $c$ is equal to 3 . 
THEOREM 5.3. The degree of a cubic geodesic immersion of $M$ into $\check{M}=$ $M^{n+p}(c)$ is equal to 3 , provided that it is proper.

Remark. We denote by $P_{m} C$ an $m$-dimensional complex projective space, and by $S_{C}^{m}$ the unit sphere in $C^{m+1} . \quad P_{m} C$ is considered as the quotient space of $S_{C}^{m}=S^{2 m+1}(1)$ obtained by identifying $x$ in $S_{c}^{m}$ with $x a$, where $a \in C$ such that $|a|=1$. The canonical Riemannian structure on $P_{m} C$ is the invariant metric such that the Hopf fibration $\pi: S_{C}^{m} \rightarrow P_{m} C$ is a Riemannian submersion. Accordingly, for an eigenfunction $f$ on $P_{m} C$ with respect to the Laplacian, so is $f \circ \pi$ on $S_{C}^{m}$ [9]. If $f \circ \pi$ is a polynomial of degree $r$ with its corresponding eigenvalue $\lambda(r)$, we have $\lambda(r)=r(2 m+r)$, and the standard immersion $\iota_{r}: P_{m} C \rightarrow S^{N(r)}(1)$ is given, where $N(r)+1=\frac{m+2 r}{m}\left\{\frac{(m+r-1) !}{r !(m-1) !}\right\}^{2}$ is the dimension of the space consisting of all hermitian harmonic polynomials of degree $(r, r)$ on $P_{m} C[1,2]$. Moreover, it is seen in $[2,8]$ that the degree of $c_{1}$ is equal to 2. As for the standard immersion $\iota_{2}$ of $P_{m} C$ into $S^{N(2)}(1)$, the degree is greater than 3 . In fact, the first normal space $N_{x}^{1}$ at any point $x$ has the dimension not greater than $2 m^{2}-m-1$, because of the characterization of complex projective spaces, and it implies $N(2)>n+\operatorname{dim} N_{x}^{1}+\operatorname{dim} N_{x}^{2}$.

Remark. Let $\iota_{0}$ be a totally umbilical immersion of $S^{N(r)}(c)$ into an $(N(r)+q)$ dimensional real space form $\bar{M}=M^{N(r)+q}(\bar{c})$, where $\bar{c}<c$. For the standard immersion $\iota_{r}$ of $S^{n}(c)$ or $P_{m} C$ it is easily seen that the degree of the composition $\iota_{0}{ }^{\circ} \iota_{r}$ of $M$ into $\bar{M}$ is equal to that of $\iota_{r}$, and the immersion $\iota_{0}{ }^{\circ} \iota_{r}$ has non-zero mean curvature.

6. Compact cases. In the rest of this paper, let $c$ be a cubic geodesic immersion of an $n$-dimensional Riemannian manifold $M$ into an $(n+p)$-dimensional real space form $\tilde{M}=M^{n+p}(c)$ of constant curvature $c$. Accordingly, any geodesic $\gamma$ in $M$ is a helix in $\tilde{M}$ and the first curvature $k$ and the second curvature $l$ are positive constant. The present section is devoted to the case where the submanifold $M$ is compact.

Now, for any point $x$ in $M$ and any orthonormal vectors $u$ and $w$ at $x$, let $\gamma_{u}$ (resp. $\gamma_{w}$ ) be a normal geodesic passing through $x=\gamma_{u}(0)\left(\right.$ resp. $\gamma_{w}(0)$ ) and let $X=\gamma_{u}^{\prime}$ and $W=\gamma_{w}^{\prime}$. Then, by virtue of (1.17), it satisfies

$$
\begin{gathered}
\widetilde{\nabla}_{X}\left(\nabla^{\prime 2} \sigma(Y, Z, U, V)\right)=\nabla^{\prime 3} \sigma(Y, Z, U, V, X)+\nabla^{\prime 2} \sigma\left(\nabla_{X} Y, Z, U, V\right)+\cdots \\
+\nabla^{\prime 2} \sigma\left(Y, Z, U, \nabla_{X} V\right)-\sum_{i}\left\langle\nabla^{\prime 2} \sigma(Y, Z, U, V),\left(X, e_{\imath}\right)\right\rangle e_{\imath}
\end{gathered}
$$

for any vector fields $Y, Z, U$, and $V$ along $\gamma_{u}$ tangent to $M$. Next, we put $u_{\imath}=u(\imath=1,2,3)$ and $u_{4}=v$ in (5.4). Then, differentiating it (resp. (5.3)) in the direction of $X$ (resp. $W$ ), and making use of (6.1), (4.8)" and the equation obtained by putting $u_{1}=e_{\imath}$ and $u_{\imath}=u(\imath=2,3,4)$ in $(4.10)^{\prime}$, we have 


$$
\begin{aligned}
& \nabla^{\prime 3} \sigma(u, u, u, v, u)=-\left(3 c+3 k^{2}+l^{2}\right) \nabla^{\prime} \sigma(u, u, v) / 2 \\
& \quad+6 \sum_{\imath}\left\{\left\langle\sigma(u, v), \sigma\left(u, e_{\imath}\right)\right\rangle \nabla^{\prime} \sigma\left(u, u, e_{\imath}\right)+2\left\langle\nabla^{\prime} \sigma(u, u, v), \sigma\left(u, e_{\imath}\right)\right\rangle \sigma\left(e_{\imath}, u\right)\right\}
\end{aligned}
$$

where $u$ and $v$ are orthonormal, and

$$
\nabla^{\prime 3} \sigma(u, u, u, u, w)+l^{2} \nabla^{\prime} \sigma(u, u, w)=0
$$

at $x$. It follows from these equations and the Ricci formula $(1.16)_{3}$ that we have

$$
\begin{aligned}
\left(9 c+9 k^{2}-l^{2}\right) \nabla^{\prime} \sigma(u, u, v)- & 30 \sum_{\imath}\left\{\left\langle\sigma(u, v), \sigma\left(u, e_{\imath}\right)\right\rangle \nabla^{\prime} \sigma\left(u, u, e_{\imath}\right)\right. \\
+ & \left.\left\langle\nabla^{\prime} \sigma(u, u, v), \sigma\left(u, e_{\imath}\right)\right\rangle \sigma\left(e_{\imath}, u\right)\right\}=0 .
\end{aligned}
$$

Making use of (6.2), we can prove

LEMMA 6.1. If the sectronal curvature has a minmal value $\delta$, then $l^{2} \leqq 9 \delta$.

Proof. We suppose that there exist a point $x$ and orthonormal vectors $u$ and $v$ at $x$ so that the sectional curvature $K(u, v)$ attains the minimal value. When we define the curvature transformation $K_{u}$ by $K_{u} w=R(w, u) u$ for any vector $w$ at $x, v$ becomes an eigenvector of $K_{u}$ with its eigenvalue $\delta$. Therefore we have

$$
(c-\delta)\langle v, w\rangle+\langle\sigma(u, u), \sigma(v, w)\rangle-\langle\sigma(u, v), \sigma(u, w)\rangle=0
$$

by the Gauss equation, from which it follows

$$
\langle\sigma(u, v), \sigma(u, w)\rangle=\left(c+k^{2}-\delta\right)\langle v, w\rangle / 3 .
$$

Combining (6.3) together with (6.2), we have

$$
\left(10 \delta-c-k^{2}-l^{2}\right)\left\|\nabla^{\prime} \sigma(u, u, v)\right\|^{2}=30 \sum_{i}\left\langle\nabla^{\prime} \sigma(u, u, v), \sigma\left(u, e_{\imath}\right)\right\rangle^{2} .
$$

Since the right hand side is non-negative, so is the left hand side.

We suppose that $l^{2}>9 \delta$. By means of this condition and (6.3), we have $c+k^{2}+l^{2}-10 \delta>0$. This and the above equation imply $\nabla^{\prime} \sigma(u, u, v)=0$. Even though we change a part of $u$ for that of $v$ in the course of this proof, we can assert the same property and we obtain $\nabla^{\prime} \sigma(u, v, v)=0$. Now, we put $u_{\imath}=u$ $(i=1, \cdots, 4)$ and $u_{\imath}=v(\imath=5,6)$ in $(4.7)^{\prime}$. Then it is reduced to

$$
2\left\langle\nabla^{\prime} \sigma(u, u, u), \nabla^{\prime} \sigma(u, v, v)\right\rangle+3\left\|\nabla^{\prime} \sigma(u, u, v)\right\|^{2}=(k l)^{2},
$$

which contradicts to the fact that $k$ and $l$ are positive. Consequently, we can prove the conclusion.

Q.E. D.

By this lemma, the following property is verified.

Proposition 6.2. For a proper cubic geodesic immersion of $M$ into $\tilde{M}=$ $M^{n+p}(c)$, if $M$ is compact, then it is of positıve curvature. 
7. Minimal immersions. In this section, we prove the following

THEOREM 7.1. For a cubic geodesic immersion of $M$ into $\tilde{M}=M^{n+p}(c)$, if it is minumal, then $M$ is locally symmetruc.

Since $\iota$ is minimal, the mean curvature vector $\eta=\sum_{\imath} \sigma\left(e_{\imath}, e_{\imath}\right) / n$ vanishes. By means of the definition of the multi-linear maps $\nabla^{\prime} \sigma$ and $\nabla^{\prime 2} \sigma$, we see also $\sum_{\imath} \nabla^{\prime} \sigma\left(e_{\imath}, e_{\imath}, u\right)=0$ and $\sum_{\imath} \nabla^{\prime 2} \sigma\left(e_{\imath}, e_{\imath}, u, v\right)=0$ for any unit vectors $u$ and $v$ at any point $x$. The last equation and (5.4) give

$$
\begin{aligned}
12 \sum_{i, j}\left\langle\sigma\left(u, e_{j}\right), \sigma\left(v, e_{\imath}\right)\right\rangle \sigma\left(e_{\imath}, e_{j}\right) \\
\quad=\left\{-3 n c+(n+4)\left(3 k^{2}-l^{2}\right)\right\} \sigma(u, v) .
\end{aligned}
$$

We define a symmetric matrix $H^{\alpha}$ of order $n$ by $H^{\alpha}=\left(h_{\imath \jmath}^{\alpha}\right)$ for a fixed number $\alpha$, and then another symmetric matrix $A=\left(A_{\beta}^{\alpha}\right)$ of order $p$ by $A_{\beta}^{\alpha}=\operatorname{Tr}\left(H^{\alpha} H^{\beta}\right)$. $H^{\alpha}$ can be regarded as a symmetric linear transformation on the tangent space defined by $H^{\alpha}=H_{e_{\alpha}}$, and $A$ as a positive semi-definite symmetric linear transformation on the normal space.

Lemma 7.2. Matrices $H^{\alpha}$ and $A$ satısfy

$$
\sum_{\beta} A_{\beta}^{\alpha} H^{\beta}=\left\{3 n c-3 n k^{2}+(n+4) l^{2}\right\} H^{\alpha} / 6 \quad \text { for any } \alpha .
$$

Proof. By the terminology of these matrices, (7.1) can be rewritten as $\Sigma_{\beta} H^{\beta} H^{\alpha} H^{\beta}=\left\{-3 n c+(n+4)\left(3 k^{2}-l^{2}\right)\right\} H^{\alpha} / 12$ for any index $\alpha$. On the other hand, the condition of isotropic immersion yields

$$
\sum_{\beta} A_{\beta}^{\alpha} H^{\beta}+2 \sum_{\beta} H^{\beta} H^{\alpha} H^{\beta}=2 k^{2} H^{\alpha},
$$

which, together with the above equation, implies (7.2).

Q.E. D.

Lemma 7.2 means that $A^{2}=L A$ and the matrix $A$ has at most two distinct constant eigenvalues 0 and $L$, where $L=\left\{3 n c-3 n k^{2}+(n+4) l^{2}\right\} / 6$. Since $A$ is positive semi-definite, the constant $L$ is non-negative. If $L=0$, then $A$ is a zero matrix on $M$, which implies that $c$ is totally geodesic. Thus, without loss of generality, we may assume that it is positive.

Proof of Theorem 7.1. For any point $x$, we consider a sufficiently small neighborhood $U$ and a normal geodesic $\gamma$ passing through $x=\gamma(0)$. Let $X=\gamma^{\prime}$. Then (7.2) is equivalent to $\sum_{\imath, j}\left\langle\sigma(Y, Z), \sigma\left(e_{\imath}, e_{j}\right)\right\rangle \sigma\left(e_{\imath}, e_{\jmath}\right)=L \sigma(Y, Z)$, where $Y$ and $Z$ are parallel vector fields along $\gamma$. Differentiating this equation in the direction of $X$ and taking account of (3.2), we see that the normal component satisfies

$$
\begin{array}{r}
\sum_{\imath, \jmath}\left\{\left(\left\langle\nabla^{\prime} \sigma\left(e_{\imath}, e_{\jmath}, X\right), \sigma(Y, Z)\right\rangle+\left\langle\sigma\left(e_{\imath}, e_{j}\right), \nabla^{\prime} \sigma(Y, Z, X)\right\rangle\right) \sigma\left(e_{\imath}, e_{j}\right)\right. \\
\left.+\left\langle\sigma\left(e_{\imath}, e_{j}\right), \sigma(Y, Z)\right\rangle \nabla^{\prime} \sigma\left(e_{\imath}, e_{\jmath}, X\right)\right\}=L \nabla^{\prime} \sigma(X, Y, Z) .
\end{array}
$$


Since this property holds true for any unit vectors at $x$, we have the following relation by virtue of the above equation at $x$ and (4.13):

$$
\begin{aligned}
\widetilde{S}_{3} \sum\langle & \left\langle\sigma\left(e_{\imath}, e_{j}\right), \sigma\left(u_{1}, u_{2}\right)\right\rangle \nabla^{\prime} \sigma\left(e_{\imath}, e_{\jmath}, u_{3}\right) \\
& =3 L \nabla^{\prime} \sigma\left(u_{1}, u_{2}, u_{3}\right)-4 \sum_{\imath, j}\left\langle\sigma\left(e_{\imath}, e_{j}\right), \nabla^{\prime} \sigma\left(u_{1}, u_{2}, u_{3}\right)\right\rangle \sigma\left(e_{\imath}, e_{\jmath}\right) .
\end{aligned}
$$

Combining this equation together with (7.2), we have

$$
\begin{aligned}
& \mathfrak{S}_{3} \sum_{\imath, j}\left\langle\sigma\left(e_{\imath}, e_{j}\right), \sigma\left(u_{1}, u_{2}\right)\right\rangle\left\langle\nabla^{\prime} \sigma\left(e_{\imath}, e_{\jmath}, u_{3}\right), \sigma(u, v)\right\rangle \\
&+L\left\langle\sigma(u, v), \nabla^{\prime} \sigma\left(u_{1}, u_{2}, u_{3}\right)\right\rangle=0,
\end{aligned}
$$

from which it follows that we have

$$
3\left\|\sum_{\imath, \jmath}\left\langle\sigma(u, v), \nabla^{\prime} \sigma\left(e_{\imath}, e_{\jmath}, w\right)\right\rangle \sigma\left(e_{\imath}, e_{j}\right)\right\|^{2}+L \sum_{\imath, \jmath}\left\langle\sigma(u, v), \nabla^{\prime} \sigma\left(e_{\imath}, e_{\jmath}, w\right)\right\rangle^{2}=0 .
$$

Since two terms of the left hand side in this equation are both of non-negative and the coefficient of the second term is positive, we have $\left\langle\nabla^{\prime} \sigma\left(e_{\imath}, e_{\jmath}, w\right), \sigma(u, v)\right\rangle$ $=0$. Because $\sigma$ and $\nabla^{\prime} \sigma$ are linear forms, it implies that

$$
\left\langle\nabla^{\prime} \sigma\left(u_{1}, u_{2}, u_{3}\right), \sigma\left(u_{4}, u_{5}\right)\right\rangle=0
$$

for any unit vectors $u_{\imath}(i=1, \cdots, 5)$ at $x$, from which we can obtain the conclusion.

Q.E. D.

By the well known properties about symmetric spaces, we have the following theorem as a direct consequence of Proposition 6.2, Theorem 7.1 and a theorem due to Sakamoto [8].

THEOREM 7.3. Let c be a cubic geodesic immersion of $M$ into an $(n+p)$ dimensional sphere $S^{n+p}$. If $M$ is compact and simply connected and is minumal, then $M$ is a symmetric space of rank one.

8. Main theorem. We shall be concerned with the following Main theorem in this paper.

THEOREM 8.1 Let $M$ be an $n(\geqq 3)$-dimensional compact simply connected Riemannian manifold and let $c$ be a proper cubic geodesic immersion of $M$ into an $(n+p)$-dimensional sphere $S^{n+p}(c)$, where $p \geqq 2$. If is minimal, then the immersion is rigid to the immersion $\iota_{0}{ }^{\circ} \iota_{3}$ of $S^{n}$ into $S^{n+p}$, where $\iota_{0}$ is a totally geodesic immersion of $S^{N(3)}(c)$ into $S^{n+p}(c)$ and $\iota_{3}$ is the standard immersion of $S^{n}$ into $S^{N(3)}(c)$.

First of all, we study about the dimension of the first normal space of the submanifold. Let $r$ be the rank of the matrix $A=\left(A_{\beta}^{\alpha}\right)$. Then it is clear that $r$ 
is the dimension of the first normal space, because of the definition of the matrix.

We use here the same notation as that in the course of the proof of Theorem 7.1. Differentiating (5.4) in the direction of $X$, and taking its normal components, we have

$$
\begin{aligned}
6 \nabla^{\prime 3} \sigma\left(u_{1}, u_{2}, u_{3}, v, u\right)= & \Im_{3}\left\{\left(-3 c+3 k^{2}-l^{2}\right)\left\langle u_{1}, u_{2}\right\rangle \nabla^{\prime} \sigma\left(u_{3}, u, v\right)\right. \\
& +\left(3 c+3 k^{2}-l^{2}\right)\left\langle v, u_{1}\right\rangle \nabla^{\prime} \sigma\left(u_{2}, u_{3}, u\right) \\
& \left.-6 \sum_{\imath}\left\langle\sigma\left(u_{1}, u_{2}\right), \sigma\left(v, e_{\imath}\right)\right\rangle \nabla^{\prime} \sigma\left(e_{2}, u_{3}, u\right)\right\}
\end{aligned}
$$

because $M$ is locally symmetric and it satisfies (7.4). Moreover, since $M$ is minimal and consequently we have $\sum_{2} \nabla^{\prime 3} \sigma\left(e_{2}, e_{2}, u, v, w\right)=0$, it follows from (8.1) that

$$
12 \sum_{\imath, j}\left\langle\sigma\left(u, e_{\imath}\right), \sigma\left(v, e_{j}\right)\right\rangle \nabla^{\prime} \sigma\left(w, e_{\imath}, e_{\jmath}\right)=\left\{-3 n c+(n+4)\left(3 k^{2}-l^{2}\right)\right\} \nabla^{\prime} \sigma(u, v, w) .
$$

Therefore we have

$$
\begin{array}{r}
12 \sum_{\imath, \jmath, k, l, m}\left\langle\nabla^{\prime} \sigma\left(e_{\imath}, e_{\jmath}, e_{k}\right), \nabla^{\prime} \sigma\left(e_{l}, e_{m}, e_{k}\right)\right\rangle\left\langle\sigma\left(e_{\imath}, e_{l}\right), \sigma\left(e_{\jmath}, e_{m}\right)\right\rangle \\
=\left\{-3 n c+(n+4)\left(3 k^{2}-l^{2}\right)\right\}\left\|\nabla^{\prime} \sigma\right\|^{2},
\end{array}
$$

where $\|$ \| denotes the length of the form. When we note that the left hand side in the above equation is non-negative, we can prove the following

LEMMA 8.2. The rank of the matrix A satısfies

$$
\frac{n(n+2)}{4} \leqq r \leqq \frac{(n+2)(n-1)}{2} .
$$

Proof. Since $\iota$ is $k$-isotropic and minimal, the square of the length of the second fundamental form is equal to $n(n+2) k^{2} / 2$. On the other hand, by the definition of $A$, we have $\operatorname{Tr} A=r L=\|\sigma\|^{2}$, where $L$ is the positive eigenvalue of $A$, so we have

$$
r=3 n(n+2) k^{2} /\left\{3 n c-3 n k^{2}+(n+4) l^{2}\right\} .
$$

Furthermore it is seen in [6] that there is the following relation between $\operatorname{Tr} A^{2}$ and $\|\sigma\|: \operatorname{Tr} A^{2} \geqq 2\|\sigma\|^{4} /(n+2)(n-1)$, and the equality is true if and only if $M$ is of constant curvature. Because of $\operatorname{Tr} A^{2}=r L^{2}=L\|\sigma\|^{2}$, we get

$$
3 n(n-1) c-3 n(n+1) k^{2}+(n-1)(n+4) l^{2} \geqq 0 \text {. }
$$

Combining the non-negativeness of the left hand side in (8.2) with (8.3) and (8.4), we obtain the inequalities.

Q.E. D.

Now, due to Theorem 7.3, we may consider the submanifold $M$ as an $n$ dimensional Riemannian symmetric space of rank one. These spaces contain only 
a sphere $S^{n}$, a complex projective space $P C^{n}(n=2 m \geqq 4)$, a quaternion projective space $P Q^{n} \quad(n=4 m \geqq 8)$ and a Cayley projective space $P C a^{n} \quad(n=16)$. Let $M^{*}$ be an $n$-dimensional compact simply connected Riemannian symmetric space of rank one. We normalize the Riemannian metric of $M^{*}$ in such a way that the sectional curvature $K(u, v)$ satisfies $1 / 4 \leqq K(u, v) \leqq 1$. We denote by $K_{u}^{*}$ the curvature transformation with respect to $u$. Then we can regard $K_{u}^{*}$ as the transformation of the orthogonal complement to the vector $u$ in $M_{x}^{*}$ at any point $x$. As is well known, distinct eigenvalues of $K_{u}^{*}$ are 1 and $1 / 4 . \quad M^{*}$ is said to be of type $s$ if the maximal eigenvalue of the linear transformation has multiplicity $s$. For each projective space, $s$ is equal to $1,3,7$ or $n-1$, according as $M^{*}$ is a complex projective space, a quaternion projective space, a Cayley projective space or a sphere.

On the other hand, since $M$ has the same situation as that of $M^{*}$, we can define the concept of the type of $M$. Concerning the type of $M$, we have

LEMMA 8.3. The type s of $M$ satısfies

$$
(n+s+1) k^{2}=(n-s-1) c,
$$

if $M$ is not of constant curvature.

Proof By the definition of the type, the linear transformation $K_{u}$ on $M_{x}$ has exactly two distinct eigenvalues and the multiplicity of the maximal one is equal to $s$. Since $M$ is not of constant curvature, the rank of $A$ is less than $(n+2)(n-1) / 2$. When we denote by $H_{\imath \imath}$ a normal vector with component ${ }^{t}\left(h_{\imath \jmath}^{n+1}, \cdots, h_{\imath \jmath}^{n+p}\right)$ for any indices $i$ and $\jmath$, it follows from Lemma 7.2 that we have $A H_{\imath j}=L H_{\imath \jmath}$. Then there exist an index $\imath$ such that $H_{i i}$ is a zero vector or distinct indices $i$ and $j$ such that $H_{\imath}$ is a zero vector, because $r=\operatorname{rank} A$ $\geqq n(n+1) / 2-1$ if it is not provided. Since the equation (6.3) holds for the maximal value of the sectional curvatures, we may consider the Riemann metric of $M$ so that eigenvalues of the linear transformation $K_{u}$ are $4 K$ and $K$, where $4 K=c+k^{2}$. Thus we can choose a suitable orthonormal basis $\left\{e_{l}, \cdots, e_{n}\right\}$ in such a way that $u=e_{1}$ and $e_{2}(i=2, \cdots, s+1)$ are contained in the eigenspace corresponding to $4 K$ and the others belong to another eigenspace. Thus we have

$$
\begin{array}{ll}
K\left(e_{1}, e_{\imath}\right)=4 K & (\imath=2, \cdots, s+1), \\
K\left(e_{1}, e_{j}\right)=K & \text { for } j \geqq s+2 .
\end{array}
$$

Accordingly, (1.7) and (1.8) imply $\sigma\left(e_{1}, e_{\imath}\right)=0$ and $\left\|\sigma\left(e_{1}, e_{j}\right)\right\|^{2}=K$. Using these results and $\sum_{i}\left\langle\sigma\left(u, e_{2}\right), \sigma\left(v, e_{2}\right)\right\rangle=(n+2) k^{2}\langle u, v\rangle / 2$, we have

$$
\sum_{\imath}\left\langle\sigma\left(e_{1}, e_{\imath}\right), \sigma\left(e_{1}, e_{\imath}\right)\right\rangle=\frac{n+2}{2} k^{2}=k^{2}+\frac{\left(c+k^{2}\right)(n-s-1)}{4},
$$

from which the necessary equality is given.

Q.E.D. 
We suppose that $M$ is not of constant curvature. It follows from (8.3) that we have $r<(n+2) k^{2} /\left(c-k^{2}\right)$, because $k$ and $l$ are positive, which together with Lemma 8.3 implies $r<(n+2)(n-s-1) / 2(s+1)$. This contradicts to Lemma 8.2. Consequently, $M$ must be a real space form. Namely, $M$ is an $n$-dimensional sphere and the degree of the immersion $c$ is 3 . Thus, we can apply Theorem 4.5 in [6] to our case, and the assertion in Theorem 8.1 is thereby proved.

Remark. We note here that the proof of Lemma 3.1 in [6] contains an error and Theornm 4.5 in the paper is true under the additional condition that the mean curvature vector $\mathfrak{h}$ is parallel in the normal bundle.

\section{BIBLIOGRAPHY}

[1] M. Berger, P. Gauduchon and E. Mezet, Le spectre d'une variete's riemannienne, Lecture notes in Math., Springer-Varlag, Berlin-Heidenberg-New York, 1971.

[2] M.P. do CARMo AND N.R. WALlach, Minimal immersions of spheres into spheres, Ann. of Math., 93 (1971), 43-62.

[3] S. L. HonG, Isometric immersions of manifolds with planar geodesics into Euclidean space, J. Differential Geometry, 8 (1973), 259-278.

[4] T. IKAWA, Remarks on circles in Riemannian geometry, to appear.

[5] J. A. Little, Manifolds with planar geodesics, J. Differential Geometry, 11 (1976), 265-285.

[6] H. NAKAgAwA AND T. ITOH, On isotropic immersions of space forms into a sphere, To appear in Proc. of Japan-United States Seminar on Minimal submanifolds, including Geodesics, 1978.

[7] B. O'Neill, Isotropic and Kaehler immersions, Canad. J. Math., 17 (1965), 909-915.

[8] K. Sakamoto, Planar geodesic immersions, Tohoku Math. J. 29 (1977), 25-56.

[9] N.R. Wallach, Minimal immersions of symmetric spaces into spheres, Symmetric spaces, Dekker, New York, 1972, 1-40.

Department of Mathematics

UNIVERSITY OF TSUKUBA

305, IBARAKI, JAPAN 